\title{
Práctica pedagógica de docentes en formación como práctica social situada*
}

Pedro Antonio Chala-Bejarano

https://orcid.org/oooo-0002-7724-4578 Pontificia Universidad Javeriana, Colombia

pchala@javeriana.edu.co

\section{Magda Rodríguez-Uribe}

https://orcid.org/oooo-0003-4291-2270 Pontificia Universidad Javeriana, Colombia rodriguez.magda@javeriana.edu.co

\section{Harold Castañeda-Peña}

https://orcid.org/oooo-0002-6828-8712 Universidad Distrital Francisco José de Caldas, Colombia hacastanedap@udistrital.edu.co

\section{Adriana Salazar-Sierra}

https://orcid.org/oooo-0001-6187-1449 Pontificia Universidad Javeriana, Colombia adriana.salazar@javeriana.edu.co

\section{Resumen}

La transferencia es un fenómeno poco estudiado en referencia al periodo de la práctica pedagógica de docentes de lenguas en formación desde marcos que la conciben como una práctica social constituida por una arquitectura. Este estudio interroga por la transferencia como una práctica social situada. Se presentan los resultados de una investigación de carácter cualitativo en la que 389 practicantes de 16 cohortes de licenciatura en lenguas destacaron en sus autovaloraciones al finalizar la práctica pedagógica. Los estudiantes realizaron su práctica como docentes de inglés y francés en una universidad privada de Bogotá (Colombia) entre 2009 y 2016. Los hallazgos del análisis coaxial de los eventos narrativos muestran que la transferencia transciende la visión dicotómica teoría/práctica y se percibe como una práctica social situada comprendida dentro de unas arquitecturas de práctica constituidas por 1) modos discursivos teóricos compartidos y modos discursivos de resistencia, 2) modos de hacer en la práctica misma como transformación a partir de la experiencia en la práctica y 3) modos de relacionarse, donde los actores curriculares participantes en la práctica tienen un rol significativo.

\section{Palabras clave (Fuente: tesauro de la Unesco)}

Arquitecturas de práctica; enseñanza de una segunda lengua; formación preparatoria de docentes; práctica pedagógica; transferencia de conocimiento.

\footnotetext{
* Este artículo surge de la investigación "Eventos narrativos significativos de profesores en formación en torno a experiencias de práctica docente en la enseñanza de lenguas extranjeras: Huellas desde la autovaloración" en la que trabajaron la Universidad Distrital Francisco José de Caldas y la Pontificia Universidad Javeriana.
}

Recepción: 15/10/2020 | Envío a pares: 15/05/2021 | Aceptación por pares: 07/06/2021 | Aprobación: 17/06/2021 


\title{
Teaching Practice of Pre-service Teachers as a Situated Social Practice
}

\begin{abstract}
Transfer has been little studied in the teaching practice period of preservice language teachers from frameworks that conceive it as a social practice with an architecture. This study looks into transfer as a situated social practice. It presents the results of qualitative research in which 389 preservice teachers from 16 cohorts of the foreign language undergraduate program provided a self-assessment at the end of their teaching practice. The students completed their practice as English and French teachers at a private university in Bogotá (Colombia) between 2009 and 2016. The coaxial analysis of narrative events found that transfer goes beyond the dichotomous theory/practice view and is perceived as a situated social practice within practice architectures comprising 1) shared theoretical discursive modes and resistance discursive modes, 2) ways of doing as a transformation from experience in practice, and 3) ways of relating, where curricular agents participating in the practice play a significant role.
\end{abstract}

\section{Keywords (Source: Unesco Thesaurus)}

Practice architecture; second language instruction; preservice teacher education; teaching practice; know-how transfer; knowledge transfer. 


\section{Prática pedagógica de docentes em formação como prática social situada}

\section{Resumo}

A transferência é um fenômeno pouco estudado quanto ao periodo do estágio pedagógico de docentes de línguas em formação a partir de contextos que a concebem como uma prática social constituída por uma arquitetura. Este estudo questiona a transferência como prática social situada. São apresentados resultados de uma pesquisa de caráter qualitativo na qual 389 estagiários de 16 turmas de licenciatura em línguas se destacaram em suas autoavaliações ao finalizar o estágio obrigatório. Os estudantes realizaram seu estágio como docentes de inglês e francês numa universidade particular de Bogotá, Colômbia, entre 2009 e 2016. Os achados da análise coaxial dos eventos narrativos mostram que a transferência transcende a visão dicotômica teoria-prática e percebe-se como uma prática social situada compreendida dentro de arquiteturas de prática constituidas por 1) modos discursivos teóricos compartilhados e modos discursivos de resistência, 2) modos de fazer na prática em si como transformação a partir da experiência no estágio e 3) modos de se relacionar, em que os atores curriculares participantes na prática têm um papel significativo.

\section{Palavbras-chave (Fonte: tesauro da Unesco)}

Arquiteturas de prática; ensino de segunda língua; formação preparatória de docentes; estágio pedagógico; transferência de conhecimento. 
En educación, el concepto de transferencia es transversal a los procesos de enseñanza y aprendizaje. Sin embargo, es un fenómeno poco estudiado en referencia a la práctica pedagógica de docentes de lenguas extranjeras en formación. En el contexto colombiano, las investigaciones en este campo han girado en torno a la reflexión como elemento esencial de la práctica pedagógica (Ariza y Viáfara, 2009; Insuasty y Zambrano, 2010; Olaya, 2018; Viáfara, 2005), creencias de practicantes sobre la enseñanza de inglés (Castellanos, 2013; Suárez y Basto, 2017), sobre sus actitudes frente a la práctica pedagógica (Morales, 2016) y, recientemente, en torno a las representaciones sociales sobre los docentes de lenguas (Reyes y Plata, 2018); no obstante, el fenómeno de la transferencia durante su período de práctica es un tema ausente en la literatura relacionada con docentes que se forman como profesores de inglés y francés en el país. La propuesta de este estudio es indagar por los aspectos de la transferencia narrados por estudiantes-profesores de inglés y francés al valorar su práctica pedagógica. Esto contribuirá a resignificar el concepto de transferencia y asumirlo como una práctica social situada, entendida como el conjunto de acciones que se presentan en un contexto en el que la convivencia humana se desarrolla de manera específica, por ser propia de ese contexto (Schatzki, 2002).

\section{Marco teórico}

En este apartado se presenta una revisión del concepto de transferencia, en dos sentidos. El primero tiene que ver con su teorización en diferentes sectores, principalmente en el productivo y recientemente en el educativo. En segundo lugar, se aborda la transferencia como una práctica social situada, desde la perspectiva de las arquitecturas de práctica.

\section{Transferencia: conceptos, medición $y$ funciones}

Tal como afirma Fleishman (1987), la transferencia es inherente al desarrollo del ser humano y ocurre en esencia cuando este se enfrenta a nuevos aprendizajes o tareas. El concepto de transferencia se ha tratado de manera recurrente en el sector de la industria (negocios y servicios) y se asocia a la efectividad de los desempeños de los trabajadores en las empresas. En este campo, la transferencia se relaciona frecuentemente con los conceptos de aplicación y eficacia (Cano, 2016; Feixas, Durán et al., 2013; Feixas, Fernández et al., 2013; Feixas et al., 2015; Mercado, García y Lozano, 2019) y, según se afirma, se hace evidente "cuando los conocimientos y habilidades ya aprendidos afectan la manera en que se aprenden y desarrollan nuevos conocimientos y habilidades" (Cormier y Hagman, 1987, p. 1). En esta mirada, trasladada a la formación docente, se entiende la transferencia como una apuesta por identificar y describir el uso de estrategias de formación planeadas y ejecutadas con el fin de mejorar los procesos de enseñanza y aprendizaje.

Desde la perspectiva del uso, la transferencia también se puede comprender como el manejo efectivo y continuado de lo aprendido (Feixas, Durán et al., 2013; Olsen, 1998). Así, con el uso frecuente de cierta habilidad, logrado con la transferencia, se espera que se genere un cambio en el comportamiento $y$ en las actitudes, que haya mayor conocimiento y que las habilidades mejoren como resultado de la transferencia del aprendizaje al ambiente laboral (Kirkpatrick, 1998). Al respecto Feixas, Durán et al. plantean que "el análisis de las acciones formativas nos arroja una tendencia predominantemente técnica, prescriptiva y concebida para el desarrollo puntual de habilidades" (2013, p. 220).

La conceptualización de la transferencia en el sector productivo ha llevado al estudio y diseño de instrumentos para su medición. Por ejemplo, el Learning Transfer System Inventory (LTSI) de Holton, Bates y Ruona (2000) busca medir no solo si la transferencia es efectiva, sino por qué. Otro ejemplo lo dan los Factores para la Evaluación de la Transferencia (FET) de Pineda et al. (en Feixas, Durán et al., 2013), que evalúan la transferencia de la formación en la 
empresa y constituyen el primer instrumento en involucrar el papel de la educación en la valoración de la transferencia del aprendizaje. A partir de su uso se ha comenzado a estudiar la validez y la efectividad de la formación que los profesionales reciben en la universidad -medidas según la transferencia que los profesionales hacen al contexto profesional- y, más recientemente, la transferencia del saber pedagógico de los docentes universitarios.

Cabe anotar que para medir la trasferencia se acude a factores que se organizan a partir de variables individuales, organizacionales y de diseño de la formación que fueron propuestas por Baldwin y Ford (1988). Así, a partir de factores correspondientes a las variables mencionadas, el estudio de Feixas, Durán et al. (2013) rastrea en el desempeño del profesor universitario español la transferencia del conocimiento adquirido durante su formación docente. Sin embargo, empiezan también a surgir estudios en los que se arguye la necesidad de considerar otros aspectos relacionados con la transferencia del aprendizaje, como la cultura universitaria (Euler, 2015) y la necesidad de profundizar en aspectos más complejos para dar cuenta del porqué de los impactos (Chalmers y Gardiner, 2015).

Las funciones de la evaluación de la transferencia son diversas. Por un lado, se encuentran estudios que indagan por la evaluación de impacto, entendido este como los cambios observados después de la intervención pedagógica, en el contexto universitario (Cano, 2016; Feixas, Durán et al., 2013; Feixas, Fernández et al., 2013; Torres, Romero y Álvarez, 2018); estudios que intentan medir la transferencia de la evaluación formativa y compartida en el contexto de la educación primaria (Molina y López, 2019); y estudios donde se mide la transferencia en términos de innovación, encontrándose trabajos como los de Pascual, López y Hamodi (2019), en los que se evalúan propuestas de transferencia de conocimiento en dos sentidos (universidad y escuela, y realidad escolar y formación universitaria) y se describen sus ventajas, inconvenientes y las respec- tivas propuestas de mejora. Otra ruta innovadora explora la transferencia del aprendizaje teniendo las herramientas digitales como mediadoras de los procesos de enseñanza y aprendizaje; los estudios se orientan particularmente hacia los Cursos en Línea Masivos y Abiertos (Massive and Open Online Courses - MOOCs) e indagan por los factores individuales del curso y del contexto que se asocian a la transferencia (Mercado et al., 2019), o evalúan la transferencia del aprendizaje a partir de cada MOOC (Napier, Huttner y Reich, 2020).

\section{La transferencia como una práctica situada con arquitectura}

Feixas et al. (2015) afirman que en el contexto de la educación superior la investigación sobre transferencia aún está en desarrollo. En el rastreo de la literatura, aunque se encuentra una investigación en Argentina sobre la transferencia de estudiantesprofesores en el área de ciencias sociales (Alquézary Buzeki, 2017), se hizo evidente que aspectos sociales, como los modos de relacionarse y la construcción de nuevos saberes a partir de lo local, han sido poco explorados en relación con la transferencia, y menos aún en cuanto al abordaje de la transferencia de los docentes de lenguas extranjeras en formación. Este artículo mira la transferencia en eventos narrativos de futuros docentes a través del filtro de las arquitecturas de práctica (Kemmis et al., 2014).

Las arquitecturas de práctica tienen su cimiento en una concepción de la educación centrada en dos propósitos fundamentales: "por un lado, formar y cultivar individuos con el conocimiento, las capacidades y el carácter para que vivan vidas buenas -es decir, vidas comprometidas con el bien de la humanidad. Por otra parte, la educación busca formar y cultivar sociedades buenas en las que procurar el bien de la humanidad sea el valor mayor" (Kemmis y Edwards, 2018, p. 2).

Con esta visión amplia de la educación, la teoría de las arquitecturas de práctica asume un carác- 
ter multidimensional (Mahon, Francisco y Kemmis, 2016) que mira la educación y la práctica profesional desde tres perspectivas: la teórica, la analítica o metodológica y la transformativa. Desde la perspectiva teórica, pretende comprender las prácticas; desde la analítica, busca explicar cómo las condiciones circundantes posibilitan o limitan las prácticas; y desde la perspectiva transformativa, procura encontrar las formas de cambiar las condiciones adversas que restringen la educación y la práctica profesional. Esta teoría emerge, de acuerdo con Mahon et al., al "problematizar la teoría de la práctica y ofrece una visión ontológica distintiva de lo que la práctica es, de cómo las prácticas se forman, son mediadas y se relacionan entre ellas" (2016, p. 2).

La práctica, de acuerdo con Kemmis et al. (2014), es una actividad social y, por tanto, humana, en la que se entrelazan tres elementos esenciales: las formas de conocer, las formas de actuar y las formas de relacionarse (sayings, doings y relatings, respectivamente). Las maneras en las que estos tres elementos se enlazan dentro de un proyecto y en un contexto situado hacen de las prácticas actividades distintivas en ese espacio y superan lo que la persona trae consigo. Adicionalmente, "abarcan disposiciones que se encuentran allí o se traen al sitio, disposiciones con las que el individuo interactúa, o sin las cuales la práctica no puede realizarse" (Mahon et al., 2016, p. 9). Según Kemmis et al. (2014), estas dimensiones, que se presentan simultáneamente en un contexto de práctica y que se constituyen en condiciones ya existentes que pueden dar pie a las prácticas o las pueden restringir, son las siguientes:

- Disposiciones culturales y discursivas: se relacionan con el lenguaje y el espacio semántico en un contexto definido y hacen posible o no la práctica en lo que se refiere a los modos de conocer (sayings). Se presenta transferencia en una práctica pedagógica cuando el practicante asume una postura pedagógica que se ve reflejada en su discurso y que comienza a configurar su identidad como profesor. Al asumir el papel de docente, adopta, indefectiblemente, un lenguaje sobre lo pedagógico propio de un profesor en ejercicio y unas visiones personales que, como encontró De Laurentis (2013), logran una aproximación al pensamiento crítico; este discurso es fruto de su reflexión y de la interacción con otros actores pedagógicos.

- Disposiciones materiales y económicas: se relacionan con los modos de hacer (doings) en tanto permiten o limitan las acciones de las prácticas en el espacio y tiempo del sitio. Un claro ejemplo de transferencia en el ámbito de la práctica pedagógica es planear una clase para contextos socioculturales específicos. Esto se realiza gracias al desarrollo crítico del conocimiento y de habilidades que se han adquirido en el periodo de formación universitaria. Sin situarse en el contexto, la transferencia podría no tener el impacto en el cultivo de la "vida buena" de la que hablan Kemmis y Mutton (2012), así como Kemmis et al. (2014).

- Disposiciones sociopolíticas: se refieren a los recursos que admiten o restringen los modos de relacionarse, no solo entre las personas, sino entre las personas y los objetos en el sitio en el que ocurre la práctica. Se puede analizar el impacto del tipo de relación que se teje entre el practicante y los docentes en servicio. Cuando el docente en formación afirma con gratitud que la retroalimentación y los consejos del profesor titular o de su tutor fueron fundamentales para un desempeño mejorado en su práctica, tenemos ante nuestros ojos un claro ejemplo de transferencia.

La teoría de arquitecturas de práctica ha sido utilizada para aproximarse a la comprensión de diversos contextos educativos. Hardy (2010) señala en su estudio que las presiones políticas, materiales y culturales para un mayor uso de las nuevas tecnologías de enseñanza fueron parcialmente responsables del estímulo y el surgimiento de 
prácticas de enseñanza productivas. Con esa misma orientación teórica, Flores (2019) ha planteado que la inducción inicial de docentes tiene condiciones de realización porque parte de las situaciones que la constituyen y que se encuentran en la red externa de apoyo que crean los mismos maestros que comienzan su práctica educativa. Es decir, la inducción como práctica no consiste simplemente en insertar a un profesor principiante en una cultura escolar desde la perspectiva laboral. Esa inserción conlleva la implementación de políticas de inducción, la socialización en la escuela y en la profesión y el apoyo a través de redes autogestionadas.

En el contexto de la educación incluyente, Hemmings, Kemmis y Reupert (2013) hicieron dos grandes hallazgos. Por una parte, encontraron que la disposición física de los edificios no solo permitió, sino también restringió el trabajo de los profesores; por otra parte, hallaron que condiciones discursivas y relacionales dieron forma a cómo los profesores pueden responder a la diversidad en sus clases y, por lo tanto, muestran la medida en que ellos pueden modelar la educación incluyente. Otros autores afirman que "cuando se comparten modos de actuar y de relacionarse en el contexto de formación de docentes de inglés en la práctica docente, se constituyen micropolíticas de acciones atravesadas por saberes compartidos con el paso del tiempo" (Castañeda et al., 2016, p. 130).

De manera análoga a los estudios descritos, basados en la teoría de arquitecturas de práctica, se argumenta que hay condiciones culturales y discursivas, materiales y económicas, y sociopolíticas que son constitutivas de la transferencia, vista como una práctica social de futuros docentes de inglés y francés. Por lo tanto, con ese piso teórico, se puede plantear que la transferencia va más allá de una aplicación de lo "aprendido", como se comprende en un número de estudios (Baldwin y Ford, 1988; Cano, 2016; Feixas, Durán et al. 2013; Feixas, Fernández et al., 2013; Feixas et al., 2015; Kirkpatrick, 1998; Molina y López, 2019; Mercado et al., 2019; Napier et al., 2020;
Olsen, 1998) y que, de manera complementaria, corresponde a un orden social (Schatzki, 2002) o práctico (Schatzki, Cetina y Savigny, 2001), anclados en condiciones materiales que existen en momentos y sitios específicos. Como se afirmó anteriormente, la literatura especializada en este tema particular es escasa para el contexto de formación inicial de docentes de lenguas extranjeras en clave de transferencia, y esta investigación pretende hacer un aporte en ese sentido.

\section{Metodología}

Teniendo en cuenta las recomendaciones de Euler (2015) y de Chalmers y Gardiner (2015), quienes sugieren considerar otros aspectos relacionados con la transferencia que complementen la tendencia a identificar factores individuales, organizacionales y de diseño de la formación, este estudio propone un acercamiento metodológico a través del análisis de eventos narrativos obtenidos en procesos de autovaloración. Se analizaron los eventos narrativos de 389 estudiantes ( $25 \%$ de ellos hombres) de un programa de licenciatura en lenguas de una universidad privada de Bogotá, Colombia, que se formaron como docentes de inglés y francés y realizaron su práctica docente entre 2009 y 2016.

Los resultados que aquí se presentan hacen parte de una investigación interinstitucional cualitativa de carácter descriptivo exploratorio que incluyó la autovaloración de estos docentes en formación. De la totalidad de participantes, aproximadamente el $25 \%$ realizó su práctica pedagógica en francés y el $75 \%$ en inglés en distintos contextos: instituciones públicas (12\%), universitarias privadas (7\%), instituciones privadas (79\%) y una fundación (2\%).

La investigación interinstitucional macro se denominó "Eventos narrativos significativos de profesores en formación en torno a experiencias de práctica docente en la enseñanza de lenguas extranjeras: huellas desde la autovaloración" y buscó identificar y caracterizar diferentes aspectos (lingüísticos, so- 
ciales, cognitivos, pedagógicos, emocionales u otros) que los futuros docentes relatan en sus autovaloraciones como significativos para su aprendizaje y que se pueden leer en Castañeda et al. (2016). A partir de las experiencias descritas por cada practicante en su autovaloración al finalizar el periodo de práctica, se definieron inicialmente categorías relacionadas con los aspectos que se quería explorar y se organizó la información en unidades hermenéuticas para su análisis. Posteriormente, se hizo una codificación axial y abierta de las autovaloraciones que resultó en la identificación de 74 códigos que fueron clasificados en 17 subcategorías organizadas de acuerdo con los aspectos que se quería caracterizar. Uno de los códigos que se identificaron en el análisis es el de transferencia, y es precisamente en el que se centra este artículo.

\section{Contexto de la práctica docente}

La práctica docente en el programa de licenciatura en cuestión se considera como el momento culminante de la formación de profesores de inglés y francés. Como asignatura, tiene dos componentes: el teórico (en forma de seminario) y el práctico, que corresponde al acompañamiento participante que hace el practicante a un docente titular en una institución durante un semestre -posterior a un periodo de observación y asistencia-. Durante este tiempo, el practicante cuenta con el apoyo de un guía pedagógico que lo acompaña de manera personalizada y lo observa en tres ocasiones en su rol de docente de lengua extranjera. El guía, los profesores titulares de la institución, el docente del seminario teórico y el practicante mismo son quienes evalúan al docente en formación tanto en su desempeño en clase como en su integración a la institución.

Parte de la evaluación incluye la autovaloración final donde el practicante, a partir de la coconstrucción de criterios de valoración con sus pares y el docente del seminario, analiza su desempeño en tres espacios: el seminario teórico, el trabajo con el guía pedagógico y la institución de práctica. De los textos de esta autovaloración es de donde se extraen los eventos narrativos de transferencia a los que se alude en este artículo y que se analizan en la siguiente sección.

\section{Hallazgos en torno a la transferencia en la práctica docente}

En los eventos narrativos analizados, son diversos los ámbitos en los que ocurre la transferencia, así como los tipos que de esta se identifican. Con base en Kemmis (2009), se examinó la transferencia en su dimensión cultural y discursiva (sayings), material y económica (doings) y sociopolítica (relatings).

\section{La transferencia en el discurso (sayings)}

En esta categoría se incluyen los factores que pueden ser parte constitutiva de una disposición discursiva (sayings). Se identifican aquí aspectos contextuales que transitan entre los saberes y las acciones que fluyen y confluyen en la práctica docente: modos de conocer que trascienden hasta el nivel del diseño curricular y llegan incluso hasta el mismo concepto de la resistencia transferencial expresada en el discurso de los practicantes.

Al analizar las experiencias significativas de los practicantes en relación con la transferencia, se encontró que los aprendizajes alcanzados en las asignaturas del área pedagógica de su programa de pregrado constituyen una parte importante de lo que están aplicando explícitamente en el contexto escolar. El componente teórico relacionado con aspectos concretos como metodologías, diseño de pruebas e innovación en el aula se ve como positivo, necesario y útil para poder desempeñarse mejor en el rol de docente. Así, se identifican eventos narrativos como el siguiente: "en relación con el alcance de las metas propuestas desde un principio, como [...] poner en práctica las metodologías y la teoría que hemos visto en nuestro proceso formativo como docentes, pienso que las cumplí y las logré llevar a cabo" (LMC-F-In-2010-1).'

$1 \quad$ La nomenclatura al final de cada extracto corresponde a las iniciales del practicante, sexo, lengua de práctica y cohorte. 
La transferencia que se puede identificar en este evento narrativo parece ser primordialmente facilitada por la formación disciplinar que la practicante recibe en la universidad y, a la vez, se percibe como una transformación del conocimiento teórico en una labor tangible dentro de un contexto situado y en una oportunidad de desarrollo profesional. En un sentido, los docentes en formación están utilizando lo que Bransford, Brown y Cocking (2000) llaman "condiciones de aplicabilidad", al tomar decisiones sobre el modo, momento, lugar y razón para utilizar explícitamente el conocimiento que adquirieron en sus clases para enfrentar situaciones que son nuevas para ellos. Por ejemplo, una practicante dice: "mi experiencia como practicante de la Licenciatura en Lenguas Modernas [en el Colegio X] fue realmente interesante y muy gratificante. Esto, debido a que aprendí muchos aspectos que había visto en clases anteriores de pedagogía, pero esta vez aplicadas a una realidad escolar" (NR-F-In-2010-1).

Desde esta perspectiva, la transferencia supone una dimensión curricular que la moldea y le da una base epistemológica propia, a partir de diversos modos de conocer, tal como lo describe NR; esto permite afirmar que, más que una transferencia estática de aprendizaje, lo que ocurre en el contexto del programa de Licenciatura es una transferencia de formación dinámica que se transforma y se actualiza constantemente con base en los conocimientos que se desarrollaron en las clases de pedagogía. Es decir, hay una apropiación del conocimiento que se manifiesta como un discurso integrado por el practicante a la realidad escolar.

Si bien la transferencia ocurre primordialmente a partir de las buenas prácticas que los docentes en formación identifican en quienes son sus modelos iniciales -los profesores titulares en el colegioen la exploración de los eventos narrativos se observa que la transferencia es un concepto mucho más amplio. Es de anotar que varios eventos narrativos muestran que hay estudiantes que también deciden no acoger desempeños con los que no están de acuerdo. Esta particular forma de resistencia crítica expresada en los modos de conocer (sayings) de los practicantes (Kemmis, 2009) se identificó como un "discurso de desaprobación" en las autovaloraciones.

Al iniciar su período de práctica, el concepto de profesor que los estudiantes han forjado durante su formación y que han ido moldeando como parte de su identidad profesional desde la teoría y desde sus propias creencias necesariamente se confronta con diferentes realidades que se viven en la institución educativa. Al reflexionar sobre la experiencia, se encuentran expresiones como estas: "durante mi estadía en el colegio traté de tomar las cosas y enseñanzas que uno puede llevar a cabo en un futuro. De igual manera, no todo lo que uno no aprende son cosas buenas" (LFM-F-In-2011-2); "me cuesta trabajo aceptar ideas sobre la educación que se oponen radicalmente a las que yo tengo, mucho más cuando no se explica por qué debe ser así y no se me da la oportunidad de argumentar mis ideas y concepciones. No obstante, mi relación con el docente titular del curso y con mis compañeras practicantes siempre fue respetuosa y profesional" (PAG-F-Fr-2010-1).

En los dos eventos narrativos, las practicantes muestran su desconcierto con respecto a lo que observan en el contexto real, en contraste con su propio conocimiento. Tal parece que ellas han ido estructurando un pensamiento pedagógico propio como parte de su identidad profesional que las lleva a reflexionar sobre sus propias prácticas y sobre las de los docentes en ejercicio, enmarcándose en lo que Mahon et al. (2016) llaman disposiciones culturales y discursivas. El segundo evento narrativo, particularmente, expresa críticas importantes a la ausencia de argumentación por parte de otros (aparentemente, el profesor titular y otros practicantes), lo cual indica una resistencia basada en la reflexión y el contraste con el sistema de creencias propio. De manera similar a lo encontrado por Insuasty y Zambrano (2010), parece ser que la práctica les ha permitido a estas estudiantes confrontar su formación como docentes con sus experiencias en un contex- 
to educativo real, lo cual también les ha permitido moldear su propia identidad profesoral. El siguiente evento narrativo incluso trasciende la crítica para incluir una mención de la acción que la practicante realiza: "logré mostrarles un modelo diferente de profesor al que tienen actualmente que solo los grita [a los estudiantes] y los trata como máquinas que producen y procesan información automáticamente" (CNR-F-In-2010-1).

El discurso de desaprobación surge, entonces, como resultado de la experiencia y sirve para expresar una resistencia transferencial que parece estar basada en lo que De Laurentis llama el "aspecto moral de las prácticas" (2013, p. 243). Tanto el rechazo de ciertas prácticas como la adopción y uso de otras (como en el caso de CNR) parecen indicar que la experiencia en el colegio contribuye a una práctica reflexiva que les permite a ciertas estudiantes hacer o evitar algunas transferencias. Las expresiones de desaprobación presentes en otros eventos narrativos analizados evidencian poca independencia y participación del practicante en su rol de docente en los contextos de práctica; es decir, una imposibilidad de desplegar el resultado de la transferencia: "debo resaltar que, aunque la institución no nos asignó trabajo más allá del acompañamiento del profesor titular, mi desempeño y propuestas de trabajo ayudaron y contribuyeron al desarrollo de la clase" (MCF-Fr-2011-2).

Para MC, la reflexión se convierte en una oportunidad para declarar posturas propias en contraste con un contexto que, desde su punto de vista, riñe con la naturaleza de la práctica. Este tipo de situaciones parece entonces generar procesos de transferencia más fluidos que dan lugar al surgimiento del pensamiento crítico y, como encontró De Laurentis en su estudio, "a reflexionar sobre el tipo de docentes que quieren ser, sobre el tipo de aula que quieren construir para sus futuros alumnos y sobre el tipo de proyecto educativo que intentan proponer" (2013, p. 245). Desde esta perspectiva, es importante analizar el papel que el docente en ejercicio tiene en el aprendizaje del docente en formación; esto debido a que se espera que el practicante asuma momentos de docencia dentro de las clases del profesor titular y que, a partir de su experiencia, incorpore la retroalimentación y la transfiera a su propia práctica, como se verá más adelante en casos exitosos.

Concluimos que la transferencia parece ser producto de la praxis y la reflexión crítica. Transferir es una práctica situada en un contexto, condicionada por situaciones específicas, y es de naturaleza social, por cuanto está influenciada por otros agentes educativos y por los conocimientos propios.

\section{La transferencia en la acción (doings)}

En esta categoría de análisis se hace referencia a los factores relativos al individuo que son constitutivos de una dimensión del hacer. Inicialmente, la transferencia que se percibe en los eventos narrativos descritos por los practicantes en relación con la acción corresponde al ámbito de la aplicación del conocimiento (Baldwin y Ford, 1988; Cano, 2016; Feixas, Durán et al., 2013; Feixas, Fernández et al., 2013; Feixas et al., 2015) o de su uso (Olsen, 1998). En este sentido, comenta una practicante: "traté de conectar la teoría con la práctica" (AAA-F-In-2014-2). Esta es una evidencia explícita de un interés personal, consciente y generalizado, que se percibe entre los profesores en formación por realizar transferencia al llevar los aprendizajes teóricos aprendidos en sus clases al contexto real en el que realizan su práctica. Sin embargo, es interesante ver que varias narraciones trascienden este nivel para abordar otras dimensiones que incluyen, por ejemplo, el valor de la reflexión sobre las temáticas tratadas en el seminario presencial de la asignatura Práctica Docente. Al respecto, una estudiante afirma:

Al inicio del proceso, tenía muchas dudas e inseguridades sobre mis capacidades como docente; sin embargo, a medida que aplicaba los conocimientos aprendidos en el seminario (y durante toda la carrera), y era mucho más reflexiva y 
analítica (con las actividades, exámenes, manejo de grupo, relaciones con los estudiantes, desarrollo cognitivo y emocional de los estudiantes, $y$ en general, de todos los procesos y acontecimientos del mundo educativo), me sentí mucho más confiada y "natural". (MCO-F-In-2009-2)

Es muy valioso que la reflexión y el análisis se mencionen como elementos significativos para enfrentar la incertidumbre que genera la novedad del contexto escolar. En este evento narrativo, los aspectos pedagógicos, metacognitivos y emocionales se narran como relevantes y se analizan a través del filtro de la reflexión individual; se evidencia aquí un fuerte impacto del modelo reflexivo característico de las asignaturas del componente pedagógico (Wallace, 1990). Además, a pesar del temor inicial planteado por MCO, la transferencia le permite alcanzar un alto nivel de confianza en sus capacidades para aplicar lo aprendido en el contexto escolar. Esto es importante, porque, como lo mencionan varios autores, un mayor sentido de autoeficacia se correlaciona con un mayor grado de transferencia. Igualmente, la experiencia de MCO demuestra que, como menciona Ditchburn (2015), una vez el estudianteprofesor deja de lado aspectos que están más relacionados con la parte técnica de la enseñanza, puede enfocarse en teorizar sobre el proceso de enseñanza y aprendizaje y no solo tener en cuenta la teoría que ha aprendido, sino establecer conexiones personales con ella; gracias a esto, sus acciones educativas adquieren una dimensión personal que moldean su identidad profesional.

Otra practicante anota: "las conclusiones del seminario [de Práctica Docente] fueron un incentivo para generar innovaciones en cuanto a la metodología y contenido de mis clases en el colegio" (AMOF-In-2012-1). Esta valoración también surge de una actitud reflexiva personal que se origina en la comparación entre lo que al parecer estaba haciendo en su rol de profesora en formación y las discusiones generadas en el seminario teórico en la universidad. Este análisis parece haberle permitido modificar su propia acción pedagógica en el contexto de la práctica docente y, como afirman Insuasty y Zambrano (2010), hacerse más consciente del valor de tomar decisiones informadas en su experiencia, en este caso con base en las reflexiones teóricas suscitadas en el seminario de práctica en la universidad. En este sentido, anota Subedi que "la transferencia se da cuando nuestros conocimientos, habilidades y destrezas afectan el aprendizaje o los desempeños de nuevas destrezas o tareas" (2004, p. 592). Sin la capacidad de autoobservación y autovaloración, y sin la evidente motivación intrínseca, probablemente no se presentaría la oportunidad de cambio, como sucedió con AMO.

A un nivel más concreto, la transferencia también se da en procesos de evaluación del aprendizaje de la lengua extranjera, tema fundamental del seminario teórico en la universidad. Por ejemplo, una practicante dice:

En él [colegio X] encontré nuevos retos para mí, los cuales me generaron mucha motivación y grandes expectativas. Gracias a esto, cada vez que tuve que enfrentarme a nuevas situaciones puse mi empeño y conocimiento para sacar adelante cualquier examen, clase o cualquier actividad en la que hiciera parte. Asi mismo, puse en práctica los temas trabajados en el seminario que me ayudaron mucho al momento de evaluar, corregir y diseñar evaluaciones. (AMZ-F-In-2009-2)

La seguridad para asumir el reto del nuevo contexto y su desempeño como profesora parece originarse en la motivación y confianza que la estudiante tiene en los temas abordados en el seminario y en el conocimiento que había adquirido previamente. Es claro que los modos de conocer, hacer y relacionarse no se desligan. AMZ percibe la importancia de "los temas trabajados en el seminario" con respecto a procesos que se relacionan con la evaluación, y realiza una transferencia asertiva al contexto escolar permeada por aspectos volitivos. Los procesos eva- 
luativos son abordados de manera similar por otra practicante: "también utilicé lo aprendido en el seminario, para ayudar a la preparación y calificación de algunos de los exámenes" (AO-F-In-2009-2).

Para estos docentes en formación, su conocimiento sobre la evaluación del aprendizaje de una lengua extranjera (testing) es significativo como parte de los procesos educativos en los están inmersos y como base de su conocimiento profesional para hacer una transferencia a su práctica docente. La transferencia incipiente en los modos de hacer percibida en estos eventos narrativos (en relación con el desarrollo de habilidades específicas ligadas con la evaluación del aprendizaje) contrasta con resultados de otras investigaciones (por ejemplo, Calik y Aytar, 2013) en las que se afirma que estas competencias solo parecen desarrollarse una vez los practicantes se convierten en profesionales en servicio.

Transferir la teoría a la práctica también parece generar una transformación personal en diferentes dimensiones como resultado de la experiencia. Esto se evidencia en los siguientes eventos narrativos: "pero a medida que transcurrió el tiempo, pude avanzar y lograr demostrar lo aprendido en los años de universidad" (ARO-F-In-2012-1); "mi proceso en el [Colegio $X$ ] ha sido la mejor experiencia que he tenido en mi formación académica y personal, no solo porque he tenido la oportunidad de poner en práctica todos mis conocimientos acerca de un área determinada, sino también porque he adquirido numerosos y gratificantes aprendizajes a lo largo del proceso" (CNR-F-In-2010-1). En estos eventos narrativos se puede ver que, al tiempo que los docentes en formación manifiestan explícitamente una transferencia de la teoría aprendida en la universidad a la práctica en el contexto del colegio, y una activación del conocimiento previo, también advierten un avance o aprendizaje personales mediante su ejercicio profesoral. Se configura, al parecer, una conciencia de que su experiencia les ha permitido evolucionar holísticamente: no solo en su quehacer, sino también en su ser.
Este proceso se percibe en otro evento narrativo en el que se identifica el valor de la observación del desempeño de un docente en ejercicio y el propio para luego transferir a un contexto determinado: "tanto la observación como la realización de clases no solo me dieron herramientas para saber enseñar, sino también formas y estrategias para manejar grupos grandes, y sobre todo si son de niños de preescolar" (YCB-F-In-2012-1). Es interesante vislumbrar aquí una reflexión consciente de la practicante sobre lo que hace en el aula, que le permite identificar cómo y qué transferir con base en su experiencia. El evento narrativo de YCB parece aportar a nuestra idea de que la trasferencia es una práctica social situada y que el contexto de la clase es en sí mismo un factor preponderante que la motiva.

En otros extractos se combinan diferentes elementos del ser humano integral (emocionales, culturales y sociales) desde los cuales se hace la transferencia hacia el desempeño en un contexto situado:

En cuanto al trabajo en la institución, es un trabajo que realizo con amor, si asi puedo llamarle. Asumi mi compromiso con responsabilidad y con la mejor disposición, lo cual se refleja en la química que se desarrolló con el resto de los profesores de mi sección; fuimos y somos un equipo desde el primer día. Gracias a esta experiencia en el [Colegio X], he tenido la oportunidad de lidiar con situaciones completamente ajenas a mi rutina diaria con una cultura que hasta hoy había sido desconocida para mí; he descubierto que esta institución es única y por tanto el manejo que le dé a dichas situaciones será determinante para mi formación como docente. En el [Colegio X] no soy solo un practicante, me han dado mi lugar y el respeto que merece mi trabajo. (LR-F-In-2011-2)

La importancia de la experiencia en el colegio parece indiscutible como parte del conocimiento profesional que LR ha formado. Sin embargo, en este evento narrativo se pueden resaltar dos particulari- 
dades: por una parte, parece indicar que la transferencia de conocimientos y habilidades está permeada por las relaciones que se pueden establecer en el contexto institucional y le da a esta práctica una dimensión social que va más allá de lo cognitivo. Y, por otra parte, la transferencia no solo ocurre de la universidad al colegio y también se puede extrapolar a la futura labor como docente en ejercicio, lo cual contribuye a moldear la identidad del profesor. La experiencia que tiene $L R$ en el colegio y la reflexión que esta genera le brinda a la estudiante las posibilidades y oportunidades que mencionan Mahon et al. (2016) para volverse un mejor practicante y realizar prácticas más sostenibles y justas que le serán útiles en su vida profesional.

Justamente, la construcción de la identidad es un aspecto primordial que se identifica en los eventos narrativos. Según Fernández, esta construcción se concibe como un proceso que se sustenta en "las propias metas y motivaciones personales, o como un proceso social de identificación colectiva con el grupo total de individuos que se dedican al mismo oficio" (2012, p. 27). Los siguientes eventos narrativos son muestra de que la práctica docente es un espacio en el cual se construye identidad: "además, apliqué lo visto en clase en la práctica y considero que lo aprendido me va a servir mucho para mi profesión como futura docente" (MMA-F-In-2010-2); "la práctica continúa brindándome nuevas alternativas [respecto] de cómo debo construir mi identidad como futura docente de lenguas extranjeras. El colegio se caracteriza por construir una relación docente-estudiante sin opresión y dogmatización alguna; entonces, logré relacionarme con los docentes de forma que podía comprender cómo se sentían ellos ante las adversidades particulares que surgían" (MJT-F-In-2016-2).

Las practicantes hacen visible la contribución de la experiencia en el colegio a la construcción de su identidad tanto profesional como personal; particularmente, en el caso de MJT, a partir de las relaciones que establece con estudiantes y profesores.
De acuerdo con Kemmis, se podría afirmar que la experiencia que adquiere un docente en formación se puede convertir en la base del conocimiento que va a transferir luego a su vida profesional. Esto "será su recurso, su identidad, su máscara, su manera, una fuente de su estatus, su forma particular de estar en el mundo y, para algunos, lo que ven como su modo de ser" (2009, p. 34).

De nuevo, la transferencia se manifiesta como una práctica que va más allá de la simple aplicación del conocimiento y que no es estática. Por una parte, no solo se transfieren conocimientos de la teoría a la práctica, sino que hay una formación y un aprendizaje profesionales que incluyen el desarrollo de habilidades docentes y una construcción identitaria que impacta el sistema de valores y creencias mediante el contacto con otros agentes educativos.

\section{La transferencia en lo social (relatings)}

Se hace referencia aquí a los factores relativos al contexto laboral constitutivos del relacionarse (relatings). Dado que el acto de transferir ocurre in situ y es social, es también indispensable analizar cómo otros aspectos presentes en la educación de los profesores en formación tienen impacto en su práctica en esta última etapa formativa. En los eventos narrativos es evidente el valor que adquieren otros actores curriculares, específicamente el profesor titular, en el contexto escolar, y el guía pedagógico, al momento de hacer transferencia, específicamente en cuanto a la construcción de conocimiento y de un perfil profesional mediante la orientación y el ejemplo. En estos eventos prevalece el modo de relacionarse, que impacta el modo de hacer y de conocer (Kemmis, 2009; Kemmis et al., 2014).

En relación con el docente titular, su rol como modelo principal de práctica parece adquirir una gran importancia, como afirma este practicante: "aprendí de ella [profesora titular] a improvisar actividades, a ser creativa, a ser un poco exigente con la indisciplina, y que así ella no llegara yo debía tener 
el salón en orden. Aprendí su manera de enseñar que, aunque no tenga un 'nombre específico', capté su manera de enseñar y luego yo en mis clases la usaba" (SCA-F-In-2012-1).

En este extracto, el rol de la docente titular como modelo de enseñanza es el punto de partida de la transferencia. La observación y el análisis permiten que la practicante reconozca como significativas las prácticas educativas de una docente con experiencia y luego transfiera ciertos aspectos a su propio desempeño. Se destacan en la cita aspectos particulares del quehacer docente que no parecen provenir de ninguna teoría, sino de la experiencia. La improvisación, la creatividad, la responsabilidad y la exigencia con la disciplina son improntas que evidencian parte de un pensamiento pedagógico que el practicante encuentra importante y susceptible de ser adoptado. Bransford et al. consideran que este proceso de apropiación de prácticas lleva tiempo, pero hay beneficios, en la medida en que el docente titular realice una '"práctica deliberada' [...] que lleva a los estudiantes a hacer seguimiento de su aprendizaje de manera que busquen retroalimentación y evalúen sus estrategias y actuales niveles de comprensión" (2000, pp. 235-236). De igual manera, es importante señalar que este tipo de transferencia es tanto situada en un contexto específico como mediada por las relaciones que se llevan a cabo en dicho contexto.

En relación con los guías pedagógicos, los practicantes los identifican como mediadores importantes en su proceso de formación para progresar en su labor como docentes. Así lo expresa esta practicante: "el trabajo de práctica en el [Colegio X] para mí ha sido maravilloso, cada día puedo poner en práctica todos mis conocimientos con la ayuda de mi guía pedagógica" (LFM-F-In-2012-2). En este caso, se evidencia la importancia del guía pedagógico como mediador en la transferencia de conocimientos, algo que otros practicantes también asocian con una causa de evolución y aprendizaje: "en cada una de las observaciones llevadas a cabo por el guía pedagógico se visualizan avances y cambios positivos tanto a nivel de lengua como en los aspectos concernientes a la clase en sí, lo que me demuestra que el feedback dado por mi guía ha sido tenido en cuenta y, por supuesto, ha sido incorporado" (MAFF-Fr-2011-2); "las reuniones con el guía pedagógico fueron un espacio pertinente donde cualquier duda era discutida y servía mucho para aclarar cosas puntuales que me ayudaron a mejorar mis clases durante las observaciones" (MA-F-In-2015-2).

Se distinguen aquí dos tipos de progreso: uno en el aspecto lingüístico (nivel de lengua) y otro en el pedagógico (relacionado principalmente con la metodología). Al respecto, como lo anotan Hattie y Timperley, existe un agente curricular-el guía pedagógico o el profesor titular, en el caso de la presente investigación- que ofrece información al practicante en lo que se refiere a su desempeño. Según estos autores, el objetivo de la retroalimentación es "reducir las discrepancias entre las comprensiones y desempeños actuales y la meta" (2007, p. 86). Como docente en formación, el practicante recibe la retroalimentación, analiza la información, la interioriza y finalmente la transfiere a nuevos y mejores desempeños que se constituyen en la meta que se quiere alcanzar.

Llama también la atención esta anotación de MFOV sobre el guía pedagógico: "además, sus observaciones fueron vitales para comprender algunas características del enfoque comunicativo, que muchas veces obviaba o que, incluso conociendo la teoría, omitía en la práctica. Sus aportes fueron punto de partida para reflexionar sobre mis prácticas" (M-Fr-2013-2). Estas omisiones al aplicar la teoría a la práctica parecen haber tenido algún efecto no deseado y llevaron al practicante, con el apoyo del guía pedagógico, a analizar su práctica y transformarla. A esto se agrega un hallazgo de Castañeda et al. (2016) en el que se identifica al guía pedagógico como fuente de conocimiento y, por lo tanto, de aprendizaje. La transferencia se origina en la interacción con el guía y a partir de la retroalimentación que este da. 
A través de su acompañamiento y retroalimentación al proceso de formación del practicante, tanto el profesor titular de curso en la institución como el guía pedagógico favorecen el desempeño del practicante: "siempre acepté el valor de la retroalimentación de la guía pedagógica y del profesor titular; hice los respectivos cambios a mejorar y siempre fue efectiva" (LM-F-In-2012-1). Si bien es posible identificar que la trasferencia se relaciona aquí con una aplicación (Cano, 2016; Feixas, Durán et al., 2013; Feixas, Fernández et al., 2013; Feixas et al., 2015; Mercado et al., 2019), es importante resaltar que esta ocurre con la mediación cognitiva de otros actores y parece ser socialmente construida a partir de maneras de relacionarse con estos actores académicos (Kemmis, 2009; Kemmis et al., 2014).

Un papel similar se identificó en la retroalimentación dada por la profesora del seminario teórico en el mejoramiento de habilidades y adquisición de saberes pedagógicos generales de los practicantes (Randall y Thornton, 2001). El siguiente evento narrativo ilustra este punto: "cumplí con las entregas de fichas, trabajos y demás, y aunque en algunas ocasiones tuve problemas aceptando la retroalimentación, siempre me esforcé en mejorar lo que me corregían y creo que eso se reflejó, sobre todo, en mi última observación" (JCB-M-Fr-1210-3). $J C B$ reconoce que el trabajo en el seminario permite una evolución en la experiencia del colegio, favorece la transferencia de conocimientos y habilidades a la práctica y contribuye a su propio mejoramiento.

Finalmente, también se identifica que la transferencia ocurre a partir de la retroalimentación entre practicantes. De manera similar a los hallazgos que describen Insuasty y Zambrano (2010) en su estudio, las discusiones entre pares que se generaron en el seminario les permitieron a los practicantes asumirse como miembros de una comunidad de práctica en la que podían intercambiar experiencias de práctica y enriquecer sus percepciones sobre la enseñanza y su desempeño docente. La interacción entre dos docentes en formación puede tener pro- pósitos de diversa índole, siendo uno de ellos el de cualificar la práctica pedagógica. Así lo demuestra este practicante:

Al comienzo no le encontraba mucho sentido a la sesión presencial [del seminario de práctica], pero luego, cuando ya tratábamos los temas desde nuestras vivencias del día a día y éramos los estudiantes quienes haciamos la clase, los temas abordados daban paso a contar no solo nuestro punto de vista, sino qué haríamos en esa situación de la que se hablaba, [respecto de] lo cual, en lo personal, hubo tips que pude aplicar $y$ en algunas situaciones me funcionaron. (MFO-F-In-2013-2)

La practicante destaca el valor de la experiencia que cada docente en formación vive como insumo para hacer ajustes en su propia práctica. Desde la perspectiva de las arquitecturas de práctica (Mahon et al., 2016), en el seminario se generan posibilidades y oportunidades para comprender la práctica profesional, mostrar cómo se facilita o se constriñe, pero, sobre todo, cómo se puede transformar con base en las experiencias vividas por los practicantes. El proceso de retroalimentación, según Díaz y Bastías (2013), se sustenta en la escucha activa y en la conversación; en ningún momento media la evaluación. En el caso particular que menciona MFO, este es un proceso de cooperación entre pares que está mediado por el contexto del trabajo en el seminario teórico en la universidad en el que se dan momentos importantes de reflexión y aprendizaje. Estas oportunidades continuas de reflexión contribuyen también a identificar lo que sucede en el salón de clases para mejorar las prácticas profesionales (Olaya, 2018).

Así, no es solo el acompañamiento por parte de profesores en servicio en la institución y de los guías pedagógicos lo que ofrece oportunidades valiosas para fortalecer la transferencia; lo es también el diálogo entre pares. Esto nos lleva a reafirmar que la transferencia es una práctica social situada que tiene lugar en un contexto mediado por la interacción 
con otros agentes pedagógicos. Es una instancia de práctica situada en la que los modos de relacionarse (relatings) parecieran determinar los modos de hacer (doings) y conocer (sayings) (Kemmis, 2009; Kemmis et al., 2014). La experiencia propia en este caso no solo se refiere a los aspectos pedagógicos per se, sino también a las dinámicas que se crean entre docentes pares y al aprovechamiento de oportunidades de discusión y reflexión entre practicantes en su rol como profesores.

\section{Conclusión}

A la luz de los hallazgos de esta investigación, es claro que la transferencia es un fenómeno en el que se conjugan múltiples dimensiones pedagógicas, todas ellas insertas en un contexto social situado donde las diferentes disposiciones de las arquitecturas de práctica permiten explicar la complejidad de dicho fenómeno. Esto amplía la comprensión de lo que se ha comprendido como transferencia, con una mirada complementaria a la propuesta y factores de medición predeterminados. Pensar en una combinación de factores y disposiciones redefine los referentes a partir de los cuales se comprenden los procesos de transferencia.

Aun cuando la práctica pedagógica se comprende como un momento culminante en la formación de futuros docentes, esencialmente es el punto de partida de la configuración identitaria del profesor. Con esto no queremos decir que el docente no cambia, pero es este el momento -cuando comienza a desprenderse de las estructuras formativas de la universidad y a ejercer su profesión-en el que transfiere de manera crítica el aprendizaje a un contexto moldeado a partir de disposiciones que permiten la transferencia, la limitan o, como se encontró, la afianzan, todo ello con la mirada crítica de un docente que tiene la capacidad de analizar el espacio y tiempo del lugar de práctica y decidir qué transfiere y de qué modo.

Estos eventos narrativos aportan a una nueva perspectiva de la formación docente cuando esta se basa en la indagación narrativa. Como afirman Huber et al. (en Porta et al., 2015), se presenta una construcción de contrarrelatos que, en el caso de estos practicantes, surgen del análisis y la reflexión. Frente a la transferencia, esto es importante, dado que permite argumentar que esta no es una aplicación mecánica, directa y acrítica de la teoría, al menos en el caso de los participantes de esta investigación. En esa línea argumental, se encuentra que la transferencia es sensible al contexto escolar e incluye resistencias discursivas y conciencia crítica que configuran la praxis y rasgos de identidad profesional. La transferencia, en el caso de la práctica docente de los profesores en formación de lenguas extranjeras de este estudio, es situada y se construye a partir de maneras de relacionarse en interconexión con formas discursivas y de hacer. Se abre así una avenida que invita a investigar más contextos de formación profesional, en clave de transferencia, entendida como una práctica social situada, de estudiantes para profesor y de docentes en ejercicio provenientes de diversos campos del conocimiento. 


\section{Referencias}

Alquézar, M.y Buzeki, M. (2017). Saber y hacer: evaluación de la transferencia de conocimientos didácticos a la práctica docente. Praxis Pedagógica, 17(20), 63-84. DOI: https://doi.org/10.26620/uniminuto.praxis.17.20.2017.63-84

Ariza, A. y Viáfara, J. J. (2009). Interweaving autonomous learning and peer tutoring in coaching EFL studentteachers. Profile. Issues in Teachers' Professional Development, 11(2), 85-104. Recuperado de: http://www. scielo.org.co/pdf/prf/v11n2/v11n2ao7.pdf

Baldwin, T. y Ford, J. (1988). Transfer of training: A review and directions for future research. Personnel Psychology, 41(1), 63-105. DOI: https://doi.org/10.1111/j.1744-6570.1988.tboo632.x

Bransford, J., Brown, A. L.y Cocking, R. R. (2000). How people learn: Brain, mind, experience, and school. Washington: National Academy Press. http://www.csun.edu/ SB4310/How\%2OPeople\%2OLearn.pdf

Calik, M. y Aytar, A. (2013). Investigating prospective primary teachers' pedagogical content knowledge of "effect of human on environment" subject in the process or teaching practice. Educational Sciences: Theory and Practice, 13(3), 1599-1605. DOI: https://doi.org/10.12738/estp.2013.3.1649

Cano, E. (2016). Factores favorecedores y obstaculizadores de la transferencia de la formación del profesorado en Educación Superior. Revista Iberoamericana sobre Calidad, Eficacia y Cambio en Educación, 14(2), 133-150. DOI: https://doi.org/10.15366/reice2016.14.2.008

Castañeda-Peña, H., Rodríguez-Uribe, M., Salazar-Sierra, A. y Chala-Bejarano, P. (2016). Eventos narrativos, comunidades y arquitecturas de práctica docente: Formación inicial de profesores de inglés y francés. Bogotá: Universidad Distrital Francisco José de Caldas.

Castellanos Jaimes, J. (2013). The role of English pre-service teachers' beliefs about teaching in teacher education programs. Profile. Issues in Teachers' Professional Development, 15(1), 195-206. Recuperado de: http://www. scielo.org.co/scielo.php?script=sci_arttextypid=\$1657-07902013000100012ylng=enytlng=en.

Chalmers, D. y Gardiner, D. (2015). The measurement and impact of university teacher development programs. Educar, 51(1), 53-80. DOI: https://doi.org/10.5565/rev/educar.655

Cormier, S. H. y Hagman, J. D. (1987). Introduction. En S. H. Cormier y J. D. Hagman (eds.), Transfer oflearning. Contemporary research and applications (pp. 1-8). Alexandria, VA: Academic Press. DOI: https://doi.org/10.1016/ B978-0-12-188950-0.50007-2

De Laurentis, C. (2013). Estudiantes narrando sus clases: relatos en el profesorado de inglés de la UNMDP. Revista de Educación, 4(6), 231-248. Recuperado de: https://fh.mdp.edu.ar/revistas/index.php/r_educ/article/ view/762/779

Díaz, C. y Bastías, C. (2013). Los procesos de mentoría en la formación inicial docente. Revista Internacional de Investigación en Ciencias Sociales, 9(2), 301-315. Recuperado de: http://scielo.iics.una.py/pdf/riics/vgn2/ vgnzaog.pdf 
Ditchburn, G. M. (2015). Remembering reflection in pre-service teachers' professional experience. Australian Journal of Teacher Education, 4O(2). DOI: https://doi.org/10.14221/ajte.2015V4On2.7

Euler, D. (2015). Mejorar las competencias docentes del profesorado universitario es necesario, pero la innovación sostenible requiere algo más. Educar, 51(1), 149-165. DOI: https://doi.org/10.5565/rev/educar.640

Feixas, M., Durán, M. M., Fernández, I., Fernández, A., García San Pedro, M. J. ... y Lagos, P. (2013). ¿Cómo medir la transferencia de la formación en educación superior?: El Cuestionario de Factores de Transferencia. Revista de Docencia Universitaria, 11(3), 219-248. DOl: https://doi.org/10.4995/redu.2013.5527

Feixas, M., Fernández, A., Lagos, P., Quesada C. y Sabaté, S. (2013). Factores condicionantes de la transferencia de la formación docente en la universidad: un estudio sobre la transferencia de las competencias docentes. Infancia y Aprendizaje, 36(3), 401-416. DOI: https://doi.org/10.1174/021037013807533034

Feixas, M., Lagos, P., Fernández, I. y Sabaté, S. (2015). Modelos y tendencias en la investigación sobre efectividad, impacto y transferencia de la formación docente en educación superior. Educar, 51(1), 81-107. DOI: https:// doi.org/10.5565/rev/educar.695

Fernández Cruz, M. (2012). Aportes de la aproximación biográfico-narrativa al desarrollo de la formación y la investigación sobre formación docente. Revista de Educación, 4(4), 11-36. Recuperado de: https://fh.mdp. edu.ar/revistas/index.php/r_educ/article/view/82/14

Fleishman, E. A. (1987). Foreword. En S. M. Cormier y J. D. Hagman (eds.), Transfer of learning. Contemporary research and applications (pp. xi-xvii). San Diego: Academic Press. DOl: https://doi.org/10.1016/B978-0-12188950-0.50005-9

Flores, C. (2019). Beginning teacher induction in Chile: Change over time. International Journal of Educational Research, 97, 1-12. DOI: https://doi.org/10.1016/j.ijer.2019.06.001

Hardy, I. (2010). Academic architectures: academic perceptions of teaching conditions in an Australian university. Studies in Higher Education, 35(4), 391-404. DOI: https://doi.org/10.1080/03075070903082334

Hattie, J. y Timperley, H. (2007). The power of feedback. Review of Educational Research, 77(1), 81-112. DOI: https:// doi.org/10.3102/003465430298487

Hemmings, B., Kemmis, S.y Reupert, A. (2013). Practice architectures of university inclusive education teaching in Australia. Professional Development in Education, 39(4), 470-487. DOl: https://doi.org/10.1080/19415257.2013.796293

Holton III, E. F., Bates, R. A. y Ruona, W. E. (2000). Development of a generalized learning transfer system inventory. Human Resource Development Quarterly, 11(4), 333-360. DOI: https://doi.org/10.1002/15321096(200024)11:4\%3C333::AID-HRDQ2\%3E3.0.CO;2-P

Insuasty, E. A. y Zambrano, L. C. (2010). Exploring reflective teaching through informed journal keeping and blog group discussion in the teaching practicum. Profile. Issues in Teachers' Professional Development, 12(2), 87105. Recuperado de: http://www.scielo.org.co/pdf/prf/v12n2/v12n2ao6.pdf 
Kemmis, S. (2009). Understanding professional practice: A synoptic framework. En B. Green (ed.), Understanding and researching professional practice (pp. 19-38). Rotterdam: Sense. DOI: https://doi.org/10.1163/9789087907327_003

Kemmis, S.y Edwards-Groves, C. (2018). Understanding education: History, politics and practice. 1 ed. Singapore: Springer. DOI: https://doi.org/10.1007/978-981-10-6433-3

Kemmis, S. y Mutton, R. (2012). Education for Sustainability (EfS): practice and practice architectures. Environmental Educational Research, 18(2), 187-207. DOI: https://doi.org/10.1080/13504622.2011.596929

Kemmis, S., Wilkinson, J., Edwards-Groves, C., Hardy, I., Grootenboer, P. y Bristol, L. (2014). Changing practices, changing education. Singapore: Springer. DOI: https://doi.org/10.1007/978-981-4560-47-4

Kirkpatrick, D. L. (1998). Evaluating training programs: The four levels. 2 ed. San Francisco: Berrett-Koehler.

Mahon, K., Francisco, S. y Kemmis, S. (2016). Introduction: Practice theory and the theory of practice architectures. En K. Mahon, S. Francisco y Kemmis, S. (eds.), Exploring education and professional practice: Through the lens of practice architectures (pp. 1-30). Singapore: Springer. DOI: https://doi.org/10.1007/978-981-10-2219-7

Mercado-Varela, M. A., García-López, R. I. y Lozano-Rodríguez, A. (2019). Aportes de un curso en Línea Masivo y Abierto (MOOC) sobre la transferencia del aprendizaje. Formación Universitaria, 12(5), 31-40. DOI: https:// doi.org/10.4067/S0718-50062019000500031

Molina, M. y López-Pastor, V. (2019) ¿Evalúo cómo me evaluaron en la facultad? Transferencia de la Evaluación Formativa y Compartida vivida durante la formación inicial del profesorado a la práctica como docente. Revista Iberoamericana de Evaluación Educativa, 12(1), 85-101. DOI: https://doi.org/10.15366/riee2019.12.1.005

Morales Cortés, Y. A. (2016). Unveiling pre-service teachers' attitudes toward teaching: The role of pedagogical practicums. Profile. Issues in Teachers' Professional Development, 18(2), 47-61. DOI: https://doi.org/10.15446/ profile.v18n2.49591

Napier, A., Huttner-Loan, E. y Reich, J. (2020). Evaluating learning transfer from MOOCs to workplaces: A case study from teacher education and launching innovation in schools. RIED. Revista Iberoamericana de Educación a Distancia, 23(2), 45-64. DOI: https://doi.org/10.5944/ried.23.2.26377

Olaya Mesa, M. L. (2018). Reflective teaching: An approach to enrich the English teaching professional practice. HOW, 25(2), 149-170. DOI: https://doi.org/10.19183/how.25.2.386

Olsen, J. H. J. (1998). The evaluation and enhancement of training transfer. International Journal of Training and Development, 2(1), 61-75. DOI: https://doi.org/10.19183/how.25.2.386

Pascual-Arias, C., López-Pastor, V. y Hamodi, C. (2019). Proyecto de Innovación Docente: la evaluación formativa y compartida en educación. Resultados de transferencia de conocimiento entre universidad y escuela. Revista Iberoamericana de Evaluación Educativa, 12(1), 29-45. DOI: https://doi.org/10.15366/riee2019.12.1.002 
Porta, L., De Laurentis, C. y Aguirre, J. (2015). Indagación narrativa y formación del profesorado: nuevas posibilidades de ruptura y construcción en la identidad docente. Praxis Educativa, 19(2), 43-49. Recuperado de: http://www.biblioteca.unlpam.edu.ar/pubpdf/praxis/v19nzao6porta.pdf

Randall, M. y Thornton, B. (2001). Advising and supporting teachers. Cambridge: Cambridge University Press.

Reyes Rincón, J. y Plata Peñafort, C. (2018). Construcción de representaciones sociales del docente de lenguas extranjeras acerca de su ejercicio. Signo y Pensamiento, 37(73). DOI: https://doi.org/10.11144/Javeriana.syp37-73.crsd

Schatzki, T. (2002). The site of the social: A philosophical account of the constitution of social life and change. Pennsylvania: The Pennsylvania State University Press.

Schatzki, T., Cetina, K. y Savigny, E. (2001). The practice turn in contemporary theory. Londres: Routledge.

Suárez Flórez, S. A. y Basto Basto, E. A. (2017). Identifying pre-service teachers' beliefs about teaching EFL and their potential changes. Profile. Issues in Teachers' Professional Development, 19(2), 167-184. DOI: https://doi. org/10.15446/profile.v19n2.59675

Subedi, B. S. (2004). Emerging trends of research on transfer of learning. International Education Journal, 5(4), 591-599. Recuperado de: https://issat.dcaf.ch/sqi/download/29846/419186/Emerging\%2otrends\%200f\%20 research\%20on\%2otransfer\%20of\%2olearning.pdf

Torres, F., Romero, L. y Álvarez, C. (2018). Transferencia de aprendizajes desde el aula formal hacia la práctica clínica: una reflexión sobre la formación de fonoaudiólogos en Chile. Revista Chilena de Fonoaudiología, 17, 1-10. DOI: https://doi.org/10.5354/0719-4692.2018.51526

Viáfara, J. J. (2005). The design of reflective tasks for the preparation of student teachers, Colombian Applied Linguistics Journal, 7, 53-74. DOl: https://doi.org/10.14483/22487085.165

Wallace, M. (1990). Training foreign language teachers: A reflective approach. Cambridge: Cambridge University Press. 